\title{
Bertrand Hervieu, Nonna Mayer, Pierre Muller, François Purseigle, Jacques Rémy - Les mondes agricoles en politique, de la fin des paysans au retour de la question agricole - Presses de Sciences Po, juin 2010, 25€
}

\section{Bernard A. Wolfer}

\section{(2) OpenEdition}

Édition électronique

URL : http://journals.openedition.org/economierurale/3108

DOI : 10.4000/economierurale.3108

ISSN : 2105-2581

\section{Éditeur}

Société Française d'Économie Rurale (SFER)

Édition imprimée

Date de publication : 30 juillet 2011

Pagination : 71-74

ISSN : 0013-0559

Référence électronique

Bernard A. Wolfer, « Bertrand Hervieu, Nonna Mayer, Pierre Muller, François Purseigle, Jacques Rémy Les mondes agricoles en politique, de la fin des paysans au retour de la question agricole - Presses de Sciences Po, juin 2010, 25€ », Économie rurale [En ligne], 324 I juillet-août 2011, mis en ligne le 18 juillet 2011, consulté le 10 décembre 2020. URL : http://journals.openedition.org/economierurale/3108 ; DOI : https://doi.org/10.4000/economierurale.3108 
Bertrand HERVIEU, Nonna MAYER,

Pierre MULLER, François PURSEIGLE, Jacques RÉMY

\section{Les mondes agricoles en politique, de la fin des paysans au retour de la question agricole}

Presses de Sciences Po, juin 2010, $25 €$

$\mathbf{L}^{2}$ a France a des rapports particuliers avec la terre et les paysans. C'est à I'analyse de ces rapports que s'attachent régulièrement, depuis plus de cinquante ans, des universitaires et des chercheurs de sciences sociales. Un premier livre, Les paysans et la politique dans la France contemporaine, a été publié en 1958 sous la direction de Jacques Fauvet ${ }^{1}$ et Henri Mendras, aux éditions Armand Colin sous I'égide de Sciences Po. Le dernier vient d'être publié, aux presses de Sciences Po, sous la direction de Bertrand Hervieu, Nonna Mayer, Pierre Muller, François Purseigle et Jacques Rémy. II a pour titre : Les mondes agricoles en politique, de la fin des paysans au retour de la question agricole.

Ces deux titres témoignent assez bien de l'évolution rapide et profonde de l'agriculture française. Le premier faisait encore des paysans le centre de la question agricole. Le second opus avait encore le mot paysan dans son titre, I'Univers politique des paysans. Le troisième avait tenu compte de l'accession des agriculteurs au rang d'interlocuteurs privilégiés du pouvoir politique. Le dernier fait sien le constat d'Henri Mendras de la fin des paysans, annoncée en 1967 et dresse de l'agriculture française, et de ceux qui la représentent, un tableau " de mondes »

1. Jacques Fauvet était chef du service politique au Monde. À noter que plusieurs journalistes ont collaboré à ce premier opus. tant ce qui était parfois nommé le " monde agricole " semble aujourd'hui divisé, éclaté en ensembles adjacents aux destinées différentes.

Sans doute faudrait-il conseiller à tous ceux qui veulent comprendre l'agriculture française de relire les trois premiers livres. Bertrand Hervieu, I'organisateur principal de ce nouveau livre, nous évite, en grande partie, cet effort en expliquant fort bien cette évolution, la replaçant dans l'évolution contemporaine de la société française. II souligne que le premier livre " insistait sur la diversité et la stabilité " ; une diversité des types de paysans, de régions, de productions ; une stabilité dans leurs appartenances, religieuse et politique, analysées en des monographies approfondies. Le second met en lumière "le changement et I'unité ". II est vrai qu'entre 1950 et 1970, en une génération donc, l'agriculture française avait connu une mutation sans précédent (analysée par Michel Gervais) une réduction rapide de la population agricole, un agrandissement des structures, la croissance élevée de la production et de la productivité. L'unité est alors plus celle du mouvement qui saisit cette agriculture qu'une réalité tangible à tout un chacun. L'analyse de Claude Servolin sur la petite production marchande qui caractérisait, à son avis, la production agricole dans le monde capitaliste, théorise en quelque sorte cette unité. Marcel Jollivet s'interrogeait de son côté sur l'existence de classes sociales dans les sociétés rurales, ce que Mendras contestait. L'unité est surtout syndicale et revendiquée comme telle, même si elle souffre de contradictions exprimées dans des luttes, et politique, l'agriculture étant l'objet d'une politique agricole forte à ce début de la $V^{e}$ République qui impulse et accompagne le mouvement de transformation. 
Le livre de 1990 insiste sur la permanence des liens entre l'agriculture et l'État: "L'agriculture est depuis toujours une affaire d'État ", malgré l'Europe et la mondialisation. Cette affirmation (que je partage peut-être imprudemment) était discutable en une période d'intégration de l'agriculture dans les négociations du GATT. Elle est devenue et ne cesse d'être depuis une pierre d'achoppement, un empêchement à conclure. Il n'est donc pas étonnant que le dernier opus s'interroge dans son ultime chapitre sur la (encore) faible consolidation du nouveau " modèle sociopolitique agricole " qu'esquissait Pierre Coulomb en 1990. Alors, les mondes agricoles ne sontils aujourd'hui qu' " en politique ", le « en » désignant une inclusion fragile, contestée ou provisoire?

« II y a cinquante ans, les agriculteurs étaient majoritaires et divers mais ils formaient un monde unifié face aux sociétés urbaines, alors qu'aujourd'hui ils sont minoritaires, segmentés et constituent définitivement des mondes pluriels. Assurément, il ne s'agit plus d'un monde mais bien de mondes agricoles. "Ainsi Bertrand Hervieu justifie-t-il, en quelque sorte, et le titre et une nouvelle figure agricole. Ni paysan ni agriculteur, pas encore tout à fait entrepreneur, mais déjà plus familial. Des mondes, donc.

La première partie, coordonnée par Jacques Rémy, montre en effet les transformations du métier, des identités professionnelles, des rapports à la nature, aux genres, à la société. Ce qui saute le plus aux yeux c'est sans doute l'éclatement des structures traditionnelles et la création de multiples façons d'être producteur agricole, qui témoignent le plus fortement à la certitude que la figure du paysan a définitivement disparu. Mais on peut aussi y voir des volontés d'affirmer des identités particulières qui distinguent chaque monde agricole des autres afin de se faire reconnaître, en particulier par les sociétés urbaines. Il ne s'agit plus de la traditionnelle opposition ville/campagne, mais d'une volonté de créer des " aventures" dans le monde rural, en qualité d'agriculteur; un peu comme si se réalisait enfin le récit de Serge Mallet, Les paysans contre le passé !

S'il semble bien établi que l'exploitation familiale, en tant que structure de production, n'est plus l'alpha et l'oméga de la production agricole, les analyses ici rassemblées n'établissent pas vraiment son remplacement par une forme "entrepreneuriale ". Sans doute manque-t-il une analyse théorique des formes de production agricole, comme I'avait faite Claude Servolin. La dimension familiale reste dominante, au moins en nombre, partout dans le monde, y compris en Amérique du Nord, sous des formes variées, certes, mais incontestables. La famille n'a-t-elle pas également beaucoup changé dans l'ensemble des sociétés ? La singularité des femmes chefs d'exploitation, étudiée par Sabrina Dahache, ouvre des voies pour analyser ces éclatements, de même que le chapitre sur les agriculteurs entre clôtures et passerelles (Dubuisson-Quellier, Giraud). Nous sortons donc de cette première partie à la fois très satisfaits des éclairages présentés et un peu frustrés devant un manque évident. Mais cela ne doit pas faire fuir, au contraire. Cela signifie qu'il y a une vraie question, bien abordée et qu'il reste du travail.

La seconde partie, sur les représentations et les modes d'actions, coordonnée par François Purseigle, poursuit les analyses, traditionnelles depuis le premier opus, sur les représentations politiques et syndicales des producteurs agricoles. Cette partie illustre bien les changements considérables aussi bien dans les appartenances politiques que 
syndicales. L'unité syndicale, emblématique des années 1950 à 1985, a perdu de son importance. De nouveaux syndicats représentent aujourd'hui des façons différentes d'être agriculteur, de se représenter, de produire et vendre, de se défendre aussi. Les analyses de Serge Cordellier et de Roger Le Guen sont, à cet égard, particulièrement pénétrantes. Elles mettent en évidence les difficultés, mais aussi, disent-ils, la nécessité qu'ont les agriculteurs à se définir en un modèle entrepreneurial. Sera-il I'avenir ? Les petits agriculteurs représentent pourtant toujours plus de $30 \%$ du total, un pourcentage dont la constance dans le temps aurait mérité un instant d'interrogation ${ }^{2}$. Ce serait une réponse à la frustration évoquée plus haut. Les élections professionnelles en sont déjà des marqueurs. Coordination rurale, Confédération paysanne sont plus particulièrement analysées dans des chapitres spécifiques. De même une histoire politique des luttes autour des OGM. L'identité professionnelle affichée par ces groupes est spécifique de leurs combats. La Fédération nationale des syndicats d'exploitants agricoles (FNSEA), et ses associations multiples, n'est plus étudiée alors qu'elle était au cœur des livres précédents : une appréciation de sa perte d'influence ou d'intérêt ? Enfin, une analyse " écologique ${ }^{3}$ du vote des agriculteurs lors de I'élection présidentielle montre des appartenances agricoles majoritairement à droite, mais assez différenciées selon les régions et les traditions religieuses ou laïques, sans qu'il y ait des automatismes: I'histoire imprime sa marque sur la longue durée.

2. Il est vrai que cette question est peu étudiée aujourd'hui. Voir cependant les recherches récentes d'étudiants de Philippe PerrierCornet.

3. À l'instar de celle réalisée par Joseph Kaltzmann dans le premier opus.
Une troisième partie place ces mondes agricoles dans leurs perspectives européennes ou mondiales. Dans son introduction, Pierre Muller estime que le monde rural n'est plus un lieu où se fabrique une explication du global. L'at-il jamais été ? N'a-t-il pas plutôt été longtemps un lieu d'inscription d'une histoire écrite ailleurs ? Ce qui aurait changé, ce sont ceux qui écrivent cette histoire, cette justification. Il est vrai que la production agricole est pensée à un niveau mondial et urbain, désincarné de la réalité de sa production. Pierre Muller met en lumière l'enjeu que représente, pour les acteurs des mondes agricoles, la perte de cette autonomie. Les chapitres précédents ont montré comment ces acteurs tentent d'y résister. Dans cette partie, d'autres façons sont examinées : la mise en place de standards de qualités, notamment, peuvent contribuer à relocaliser les productions, comme ils peuvent aussi échapper aux producteurs. Les lieux de luttes deviennent en partie immatériels, comme l'époque en produit tant! Les enjeux de la PAC, joués au niveau international dans le cadre des négociations de I'OMC (Doha round) sont analysés par Tancrède Voituriez et Benoît Daviron comme une lutte entre des libéraux du marché concurrentiel et des antilibéraux vaguement protectionnistes, ces derniers justifiés par des économistes " mécréants ${ }^{4}$, jeux auxquels participent avec plus ou plutôt moins de bonheur les OPA et les ONG françaises. Me sentant personnellement du côté des économistes mécréants (comme Voituriez et Daviron semble-t-il), j'aurais apprécié que leur conclusion soit plus percutante, moins nuancée par leur prudence et leur humilité, d'autant que si cette position française est apparemment minoritaire, elle résonne avec d'autres positions de

4. Dont Krugman serait un modèle acceptable et Allais une référence refusée. Deux Nobels. 
grands pays agricoles du monde si l'on observe la réalité des négociations et surtout des échecs de celles-ci.

Enfin, Marielle Berriet-Selliec et Aurélie Trouvé analysent les évolutions régionales et européennes des politiques. Les politiques agricoles ne sont plus seulement globales ou sectorielles, elles s'affinent dans des contextes locaux ou régionaux depuis la réforme de 1992. Ces politiques sont-elles des variables d'ajustement des tergiversations des politiques agricoles entre leurs dimensions européennes et/ou nationales ? Dans le cadre de la libéralisation des marchés, la régionalisation des politiques peut à la fois autoriser une meilleure adaptation des politiques aux réalités territoriales et justifier certaines pratiques au nom du développement, contre la critique du soutien. Mais ce nouveau modèle sociopolitique esquissé en 1990 (voir ci-dessus) reste dominé par des impératifs nationaux ou européens.

Je ne peux que recommander la lecture de cet ouvrage. On ne partagera pas toujours dans le détail certaines analyses et conclusions. Mais il est justement fait pour susciter débats et controverses, et mieux, relancer certains travaux. En cette période de relative disette d'ouvrages d'analyse des agricultures et de leurs politiques, il s'impose. Il est de son temps. On remarquera qu'il est peu fait mention de luttes, comme il y avait autrefois. Pourtant c'est une caractéristique des mondes agricoles. Il est vrai que ces luttes avaient décru entre 1995 et 2008 , sauf sur les OGM. Mais, depuis 2008, les grèves du lait, les tensions sur les céréales et l'élevage, l'émergence de nouvelles formes de luttes, au niveau européen parfois, devrait susciter un nouveau livre. La mise en critique de l'agriculture par les acteurs et consommateurs urbains mériterait également une analyse sérieuse. Espérons que cela ne sera pas dans trop longtemps. Et pourquoi ne pas envisager des comparaisons européennes et internationales, comme contrepoints ou miroirs?

Car il y a bien retour d'une question agricole. Et sans doute pour longtemps. Elle avait simplement été oubliée dans les îles paradisiaques du commerce libéré et de l'abondance automatique des économistes heureux. Un retour à la réalité ne peut que faire du bien.

Bernard A. WOLFER Directeur de recherche, MONA, INRA 